\title{
Civilisations
}

Revue internationale d'anthropologie et de sciences

humaines

$41 \mid 1993$

Mélanges Pierre Salmon II

\section{Le journal des voyages au pays du caoutchouc rouge}

\section{Gabriel Thoveron}

\section{OpenEdition}

\section{Journals}

\section{Édition électronique}

URL : http://journals.openedition.org/civilisations/1712

DOI : 10.4000/civilisations. 1712

ISSN : 2032-0442

\section{Éditeur}

Institut de sociologie de l'Université Libre de Bruxelles

\section{Édition imprimée}

Date de publication : 1 septembre 1993

Pagination : 279-289

ISBN : 2-87263-094-5

ISSN : 0009-8140

\section{Référence électronique}

Gabriel Thoveron, «Le journal des voyages au pays du caoutchouc rouge », Civilisations [En ligne], 41 | 1993, mis en ligne le 29 juillet 2009, consulté le 02 mai 2019. URL : http:// journals.openedition.org/civilisations/1712 ; DOI : 10.4000/civilisations.1712

Ce document a été généré automatiquement le 2 mai 2019.

(c) Tous droits réservés 


\title{
Le journal des voyages au pays du caoutchouc rouge
}

\author{
Gabriel Thoveron
}

Devant la bouquinerie, des auteurs passent, comme des animaux fabuleux. Voici André Gide. Voici Mac Orlan. Voici Salmon.

Pierre Véry, Léonard, ou les délices du bouquiniste

1 Que de moments agréables j'ai partagés avec Pierre, bouquinant sur le vieux marché, ou chez Monsieur Henry à l'enseigne de Philo, ou ailleurs encore. Ses avis de bibliophile averti sont toujours très précieux. Nous ne cherchons pas les mêmes livres, mais chacun connaît les goûts de l'autre, et peut lui signaler une possible trouvaille. Nous ne cherchons pas les mêmes livres, et pourtant il m'arrive, dans l'un ou l'autre ouvrage de fiction, de découvrir une page pouvant l'éclairer, sinon sur l'Histoire de l'Afrique, du moins sur la manière dont elle se racontait, à certains moments, dans le reste du monde. Il n'est pas indifférent qu'Edgar Rice Burroughs écrive, en 1912, dans le premier volume des aventures de Tarzan, Tarzan of the Apes, que la cruauté des anthropophages était liée au souvenir de "toute la barbarie dont avaient fait preuve à leur égard les officiers blancs de Léopold II de Belgique - cet hypocrite sans égal -" et de la manière dont eux, "derniers survivants d'une tribu autrefois riche et puissante, avaient dû fuir l'Etat libre du Congo"1 ; le romancier évoquait une colonisation qui n'hésitait pas à largement user de la chicotte, mais faisait aussi écho à la rage d'un clan anglo-américain qui n'imaginait pas que le monde puisse se civiliser autrement qu'en langue anglaise. Conan Doyle, génial inventeur de Sherlock Holmes, mais par ailleurs "ganache victorienne (...) suppôt de l'Empire" selon certains parmi les plus fervents de ses admirateurs français ${ }^{2}$ pouvait à la fois dénoncer, sous les applaudissements de Winston Churchill, The Crime of the Congo, et justifier The War in South Africa. Racontant La vie de Sir Arthur Conan Doyle, John Dickson Carr, tout en qualifiant Léopold II de façon nuancée, "vieux satrape endurci dont le cynisme n'était pas dépourvu de bienveillance", reconnaît lui-même que la Grande-Bretagne n'était, en cette affaire, "pas tout à fait désintéressée : les Anglais désiraient le libre échange aussi bien qu'un peu d'humanité"3. Un peu d'humanité, beaucoup de libre échange. 
2 Que pensait-on généralement du Congo en France ? Sans doute ce qui se pouvait lire dans Le journal des Voyages, dont le titre était apparu en 1877, et où les adolescents qui trouvaient trop mièvres les publications pour enfants sages qui leur étaient alors destinées, allaient chercher de pleines brassées d'aventureuses émotions. Pierre Versins a dressé en une phrase - assez longue il est vrai - le portrait de cet étonnant hebdomadaire : "Raciste, sadique, chauvin, crédule jusqu'à l'imbécilité, flattant les pires instincts de la Bête, faisant de l'image (ô les couvertures de Castelli, et les doubles pages centrales suant la haine et la cruauté !) le support même du subconscient de l'homme asservi par sa merde intérieure, bref, tout pour plaire, mais merveilleux par l'imagination et riche de presque tout ce que la littérature populaire pouvait offrir à l'époque, Le journal des Voyages et des Aventures de Terre et de Mer est peut-être le seul périodique au monde à avoir écrasé trois-quarts de siècle de sa magnificence un peu sulfureuse"4. Un jugement excessif dans la réprobation autant que dans l'éloge : mais l'hebdomadaire comportait à la fois une partie littéraire où les meilleurs épigones de Jules Verne se retrouvaient, offrant d'imaginatifs récits d'anticipation ou d'aventureuse exploration, et une partie documentaire, celle qui va retenir notre attention ici, bien qu'elle ne soit pas toujours la mieux informée. "Louis Boussenard, nous rappelle Denise Escarpit, après avoir longtemps collaboré au Journal des Voyages dans lequel il écrit des reportages de pays qu'il n'a jamais visités (...) se met à voyager et publie des romans dont l'arrière-plan est constitué par les pays qu'il connaît..."5. Qu'importe d'ailleurs aux jeunes lecteurs qui se passionnent pour cette publication moins agressivement pédagogique que le Magasin d'Education et de Récréation d'Hetzel, mais qui tout de même peut être avantageusement montrée aux parents : on y apprend des choses.

Boussenard, dans Voyages et Aventures de Mlle Friquette, nous décrit l'enthousiasme de son héroïne : "Trois sous par semaine, qu'elle portait d'une traite à la marchande de journaux, en lui disant d'une voix émue: Le Journal des Voyages, s'il vous plait madame. Car Mademoiselle Lili ne pouvait plus attendre la mise en volume des histoires (...). Elle voulait les avoir de suite encore toutes chaudes, des presses de l'imprimerie...".

4 La vision du monde qu'elle peut en retirer, nous le voyons avec le recul, est singulièrement faussée, mais répond sans doute, à cette époque, au sens commun. D'où l'intérêt d'y aller voir.

5 Nous disposions des numéros compris entre le 9 octobre 1904 et le 26 avril 1908, nous y avons cherché ce qui se rapportait à l'Etat libre du Congo. Quantitativement plutôt maigre, notre moisson nous semble refléter assez bien l'orientation générale du Journal des Voyages: hors du monde des hommes blancs, on ne trouve guère que de drôles de gens, ayant de drôles de moeurs, des sauvages, puisqu'il faut les appeler par leur nom, en tout cas des inférieurs, et qu'il est fort urgent d'aller civiliser.

6 Sur le territoire du Congo, la bizarrerie physique est celle surtout des pygmées "si étranges que l'on a peine à croire à leur existence". Il y a les Batoua, il y a les Mamboutti, ces derniers découverts par Stanley et largement décrits dans le $\mathrm{N}^{\circ} 446$ du 18 juin 1905 : "La tribu mamboutti, composée de personnages d'un mètre ou à peine davantage, semble la moins sauvage des familles naines. Tandis que les Batoua sont affreux, trapus, très velus, presque noirs et semblables de loin à de petits gorilles, les Mamboutti ont la peau jaune clair, le front découvert, les yeux beaux et grands, l'air franc". Mais gare à leurs flèches empoisonnées : "l'homme ou la bête atteints (...) sont perdus". Or les Mamboutti, "se considérant comme les ennemis de leurs voisins (...) n'attendent pas toujours d'en 
être attaqués: au contraire, ils les provoquent souvent, par la rapine et les pillages organisés".

7 Les "sauvages" que la nature n'a pas créés petits ou laids peuvent se rendre d'eux-mêmes affreux ; ainsi de la négresse à plateau katangaise dont la photo figure dans le $\mathrm{N}^{\circ} 558 \mathrm{du}$ 11 août 1907 : "Les lignes du menton ont disparu ; le nez semble s'être élargi, les joues se sont creusées : la défiguration est complète ! Et le crâne, rasé de près, ajoute à la laideur de notre Vénus congolaise. Nous aimons à espérer que cette barbare pratique disparaîtra avant peu du Congo".

8 Il y a pire. Dans le $\mathrm{N}^{\circ} 475$ du 7 janvier 1906, on décrit la façon de se débarrasser des Ancêtres encombrants: "Quand un vieillard est devenu impotent, sa famille lui administre un narcotique, puis le coud dans un sac fait avec des peaux d'antilope, et le transporte à bonne distance du village, pour le déposer sous les buissons non loin d'un sentier. Le premier indigène qui passe feint de prendre le ballot pour une antilope et le perce de sa sagaie. Les parents, qui se tenaient cachés non loin de là, accourent en poussant des cris, et tous ces braves gens, y compris le meurtrier, déplorent le fatal 'accident' et se lamentent sur la 'mort prématurée' du vieillard".

Il y a pire. Il y a les anthropophages, tels ceux qui mangèrent l'explorateur Hodister. Ce dernier souffrit "toutes les tortures morales et physiques. Un de ses tourmenteurs à face de gorille, plus fort et plus raffiné que les autres, imagina joyeusement de lui couper d'abord les deux mains et les deux pieds que l'on fit immédiatement griller sur un brasier préparé à cet effet en face du supplicié. L'invitant ensuite à partager son ignoble repas, le même anthropopithèque, à l'aide d'une baguette de bois pointue et durcie au feu, lui introduisit de force dans la bouche des lambeaux brûlants de sa propre chair (...) l'explorateur Hodister mourut, comme le loup de Vigny, sans un cri, sans un mot. Sa dernière pensée fut pour la Belgique, sa patrie. Peut-être qu'en ce nouveau calvaire il pardonna aussi à ces bourreaux inconscients qu'il s'était proposé, comme tant d'autres, d'instruire et d'arracher à la barbarie". Cet atroce récit, titré Mangé vivant ! remplit trois pages du $N^{\circ} 591$ du 29 mars 1908, trois pages dont la Une, toute consacrée à un dessin représentant le supplice.

On le voit, Le Journal des Voyages s'adresse à des lecteurs avides de sensations fortes.

Les 8 et 15 avril 1906, il va donc consacrer une partie importante de sa surface, et chaque fois ses deux pages centrales illustrées de dessins, à un article intitulé Le Pays du Caoutchouc rouge, "rouge du sang des malheureux indigènes qui devaient le fournir aux Européens !"6. Les informations sont présentées avec beaucoup de précautions, bien que le titre - sensationnel semble indiquer clairement l'orientation du texte. L'hebdomadaire prend d'abord ses distances vis-à-vis des accusations anglaises : "Les documents que nous analysons et les illustrations que nous reproduisons viennent d'outre-Manche. C'est sous cette réserve que nous les enregistrons ici, et nous ne nous rendons pas juges des accusations que nous ne pouvons cependant passer sous silence". Mais "une commission d'enquête envoyée de Bruxelles a reconnu la réalité de quelques-uns (sic) et la défectuosité du système qui les a produites" et son rapport est rempli de faits qui, comme les témoignages produits par les Anglais, "donnent l'impression d'un spectacle d'horreur". Ce sont quasi essentiellement les documents fournis par la Commission que l'article, signé René Thierry, va citer, ce sont les témoignages recueillis par la commission qui vont faire l'objet d'illustrations dont les légendes sont significatives : "Quatre solides gaillards empoignaient le délinquant et l'étendaient à terre, un cinquième, armé de la chicotte, lui administrait une sanglante fouettée" ; "Ils avaient vu tuer leurs parents sous 
leurs yeux" ; "Lomboto amena au bateau la malheureuse femme Boachi à laquelle les soldats avaient coupé le pied pour s'emparer de l'anneau qu'elle portait"; "L'un des soldats indigènes avait saisi un enfant et lui avait brisé la tête sur un tronc d'arbre" ; "Après avoir assassiné le chef Isékéfasou, les soldats se précipitèrent sur les femmes et les enfants et les égorgèrent"...

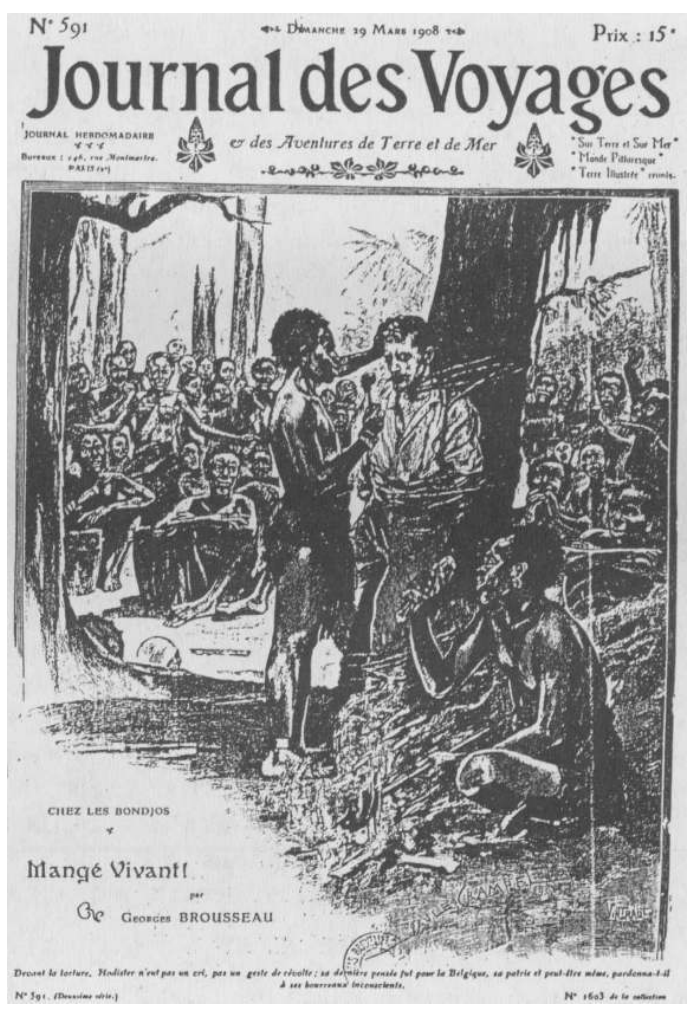

12 Il y a donc une étrange contradiction entre le début du texte, annonçant qu'il faut se méfier des témoignages évoqués, parce qu'ils sont anglais, et le corps de l'article qui se rapporte quasi exclusivement à ce qu'a, semble-t-il, authentifié la Commission d'Enquête, et que confirmeront finalement "une série de décrets du roi-souverain" qui a introduit "d'importantes réformes dans l'administration congolaise", réformes visant manifestement à éliminer les abus. Comme cela n'offre pas au lecteur les horribles sensations qu'il attend, cette information, le 4 novembre 1906, n'occupe qu'un entrefilet dans le supplément du journal, Sur Terre et sur mer - le mois géographique, tout comme l'annonce, le 2 décembre de la même année, de la création d'une école de médecine tropicale à Bruxelles.

13 Au moins les réformes permettent-elles de parler des excès au passé. Dans le $\mathrm{N}^{\circ} 570$ du 3 novembre 1907, un article illustré, titré $\mathrm{Du}$ caoutchouc ou des otages, évoque le régime auquel avaient été soumis les Africains : "A la traite avait succédé l'abus du travail forcé imposé aux indigènes. C'est au Congo surtout que cette barbare habitude était implantée (...). Il y eut des choses pires. Le Journal des Voyages les a déjà dites, et il a enregistré ici, en essayant d'en atténuer l'exagération, le long cri d'horreur soulevé notamment en Angleterre par les lugubres récits de pillages et de répressions sanglantes...". Il reste aujourd'hui le "système des otages qui choque moins le sentiment des civilisés que celui des mains coupées et des exécutions sommaires (mais qui) est encore excessif pour l'humanité d'aujourd'hui": des chefs de famille étaient détenus jusqu'à ce que des quantités suffisantes de caoutchouc aient été fournies. "Rendons cette justice à nos 
voisins les Belges qu'ils mettent tout en oeuvre pour mettre fin à cette forme nouvelle de traite...".

D'ailleurs, lit-on dans un article paru dans le $\mathrm{N}^{\circ} 561$ du 1er septembre 1907, "nous nous sommes bien gardés de nous mêler" à la campagne critiquant l'administration de l'Etat indépendant du Congo. "C'est surtout dans le domaine de la politique coloniale que les bons conseilleurs ne sont pas les meilleurs payeurs!". Evidemment, "il est incontestable que le nègre qui n'a pas encore eu un long contact avec la civilisation est (règle générale qui comporte assurément des exceptions) un grand enfant qu'il convient de mener sévèrement. Quelques mots sur les photos inédites reproduites sur cette page feront mieux comprendre notre pensée, qui n'a rien de malveillant envers la race africaine". L'article, intitulé Le Service pénitentiaire an Congo, est illustré par une image montrant une administration de chicotte : mais "qui prétendra que l'Angleterre, que les Etats-Unis sont des pays sauvages? Or, on fouette les malfaiteurs en Angleterre. Et on les fouette aussi aux Etats-Unis...". Une autre photo montre deux hommes enchaînés. "Certes, notre sensibilité souffre de voir ces deux malheureux ainsi traités. Mais que dirions-nous, si nous les voyions enfermés dans un cachot étroit, privés d'air et de lumière, comme cela se pratique trop souvent dans les colonies anglaises de l'Afrique (...). Bien qu'enchaînés pour un simple délit, ces deux noirs peuvent encore profiter de l'air et de la lumière". Une troisième photographie montre deux femmes, nues et chargées de chaînes, otages gardées jusqu'au moment où leurs maris, qui refusaient de travailler, reviendront à leur tâche. "Le moyen réussit (...). Que la nation coloniale exempte de tout péché aussi léger jette aux Belges la première pierre !"

Une ligne éditoriale cohérente se dégage finalement de ce qui semble par moment hésitant, voire contradictoire. Commercialement, le Journal des Voyages exploite le goût du public pour ces "horribles détails" qui ont fait longtemps le succès des feuilles occasionnelles, des "canards", tués par la presse populaire dans la seconde moitié du dixneuvième siècle ; politiquement, cet étalage de curiosités, qui va de l'affreux au ridicule, sert à démontrer l'infantilisme de populations qu'il convient donc d'éduquer, s'il le faut contre son gré, s'il le faut par la force. On relativise donc les manières un peu brutales, on les justifie.

$16 \mathrm{Au}$ moins convient-il que les colonisateurs ne partagent pas la cruauté des indigènes qu'ils civilisent. "Même quand nos troupes doivent employer la force contre les indigènes, elles ne se départissent point des règles d'humanité et l'effusion de sang est toujours aussi limitée que possible, lit-on dans le $\mathrm{N}^{\circ} 429$ du 19 février 1905 ; c'est la raison pour laquelle, après la collision, les vaincus indigènes deviennent toujours nos amis. Les Allemands n'agissent pas ainsi...". Les peuples n'ont pas tous les qualités de coeur françaises. Les excès de certains sont cependant gênants dans la mesure où ils risquent de jeter le discrédit sur l'ensemble de l'oeuvre coloniale : or il n'est pas toujours possible de les taire.

17 Ainsi les exactions commises dans le pays du caoutchouc rouge ne peuvent être dissimulées : la campagne menée par l'Angleterre a été trop bruyante. Il serait d'ailleurs regrettable de ne pas exploiter des illustrations et des documents qui flattent le goût sadique de nombreux lecteurs. Il convient donc de manifester pour une fois de l'esprit critique et d'émettre quelques réserves. Jusqu'au moment où l'on pourra enfin parler de ces crimes au passé et conclure leur rappel par une note optimiste: "Sans se laisser émouvoir par les protestations de leurs adversaires d'outre-Manche, les Belges sauront donner aux noirs du Congo un régime de travail qui respecte leur liberté et leurs droits 
(...). On ne verra plus (...) de longues caravanes de malheureux (...) porter un tribut à l'homme blanc, souvent aussi détesté et toujours redouté par ces humanités inférieures que le négrier ou le conquérant" (3 novembre 1907).

Etre redouté, c'était prudent, mais les civilisateurs européens voulaient aussi que l'homme blanc soit aimé par ces "sauvages", ces "barbares", ces "humanités inférieures", voire ces "anthropopithèques". De ce point de vue, l'action des agents de Léopold II au Congo avait mauvais genre, les nouvelles la concernant faisaient le plus détestable effet sur les opinions publiques : il était d'abord important de les relativiser, urgent ensuite d'annoncer que tout était rentré dans le bon ordre colonial...

\section{NOTES}

1. Tarzan, le Seigneur de la Jungle, Paris, Edition Spéciale, 1970, traduction française, p. 178.

2. Jacques Baudou et Paul Gayot, Sherlock Holmes Memorial, Paris, Clancier-Guénaud, 1982, p. 10.

3. Paris, Robert Laffont, 1958, traduction française, p. 323.

4. Encyclopédie de l'Utopie et de la Science fiction, Lausanne, l'Age d'Homme, $2^{\mathrm{e}}$ édition, 1984, p. 477.

5. La Littérature d'Enfance et de Jeunesse, Paris, PUF, Que sais-je, $N^{\circ} 1881,1981, \mathrm{pp} .86-87$.

6. $\mathrm{N}^{\circ} 488$, pp. 319 à $321 ; \mathrm{N}^{\circ} 489$, p. 335 à 338.

\section{RÉSUMÉS}

The study of literature written for the working classes highlights the common stereotypes existing at a given point in time, notably on African populations. On the basis of texts published between 1904 and 1908 in "Le Journal des Voyages", it can be proven to what extent the image imposed of the Congolese is generally awful or ridiculous. This period corresponds to the time when accusations were made by Great Britain on the way in which the collection of rubber was carried out for Leopold II's officials. "Le Journal" adopts an ambiguous attitude : the treatment of information is sensational (the public's taste for horrible details is flattered) and their comments are nuanced (it does not aim to contribute to an overall criticism of colonial activities).

\section{AUTEUR}

\section{GABRIEL THOVERON}

Faculté de Philosophie et Lettres - Université Libre de Bruxelles - Belgique 